\title{
A note on path factors of $(3,4)$-biregular bipartite graphs
}

\author{
Carl Johan Casselgren \\ Department of Mathematics \\ Umeå University \\ SE-901 87 Umeå, Sweden \\ carl-johan.casselgren@math.umu.se
}

Submitted: Dec 20, 2010; Accepted: Nov 1, 2011; Published: Nov 14, 2011

Mathematics Subject Classification: 05C70 (05C15, 05C38)

\begin{abstract}
A proper edge coloring of a graph $G$ with colors $1,2,3, \ldots$ is called an interval coloring if the colors on the edges incident with any vertex are consecutive. A bipartite graph is $(3,4)$-biregular if all vertices in one part have degree 3 and all vertices in the other part have degree 4. Recently it was proved [J. Graph Theory 61 (2009), 88-97] that if such a graph $G$ has a spanning subgraph whose components are paths with endpoints at 3 -valent vertices and lengths in $\{2,4,6,8\}$, then $G$ has an interval coloring. It was also conjectured that every simple $(3,4)$-biregular bipartite graph has such a subgraph. We provide some evidence for this conjecture by proving that a simple $(3,4)$-biregular bipartite graph has a spanning subgraph whose components are nontrivial paths with endpoints at 3-valent vertices and lengths not exceeding 22.
\end{abstract}

Keywords: path factor, biregular bipartite graph, interval edge coloring

\section{Introduction}

We use [7] for terminology and notation not defined here and consider finite graphs only. $V(G)$ and $E(G)$ denote the sets of vertices and edges of a graph $G$, respectively. In this paper, "graphs" may have multiple edges whereas "simple graphs" do not have any multiple edges.

A proper edge coloring of a graph $G$ is called an interval coloring if the colors on the edges incident with any vertex of $G$ are consecutive. An interval coloring of $G$ with colors $1,2, \ldots, t$ is called an interval $t$-coloring if at least one edge is colored $i$, for $i=1, \ldots, t$.

The notion of interval colorings was introduced by Asratian and Kamalian [4] (available in English as [5]), motivated by the problem of finding compact school timetables, that 
is, timetables such that the lectures of each teacher and each class are scheduled at consecutive periods. Generally, it is an $\mathcal{N} \mathcal{P}$-complete problem to determine whether a given bipartite graph has an interval coloring [21]. Nevertheless, trees [14, 18], regular and complete bipartite graphs [14, 18], doubly convex bipartite graphs [18], grids [11], and simple outerplanar bipartite graphs [10,6] all have interval colorings. Giaro [9] showed that one can decide in polynomial time whether bipartite graphs with maximum degree 4 have interval 4-colorings.

A bipartite graph with bipartition $(X, Y)$ is called $(a, b)$-biregular if all vertices of $X$ have degree $a$ and all vertices of $Y$ have degree $b$. The investigation of the existence of interval colorings of $(a, b)$-biregular bipartite graphs was initiated by Hansen [14]. He proved that $(2, b)$-biregular bipartite graphs have interval colorings when $b$ is even. This was extended to all $b$ by Hanson, Loten and Toft [15] and independently by Kostochka [19]. Only a little is known about $(3, b)$-biregular bipartite graphs. It follows from the result of Hanson and Loten [16] that no such graph has an interval coloring with fewer than $3+b-\operatorname{gcd}(3, b)$ colors, where gcd denotes the greatest common divisor. Asratian and Casselgren showed in [3] that the problem to determine whether a $(3, b)$-biregular bipartite graph has an interval $b$-coloring is $\mathcal{N} \mathcal{P}$-complete in the case when 3 divides $b$. This implies that there are $(3, b)$-biregular bipartite graphs without interval $b$-colorings. However, it is still an open question whether all $(a, b)$-biregular bipartite graphs have interval colorings (using any number of colors). It is conjectured that all such graphs have interval colorings (see [17]); the first open case is $(a, b)=(3,4)$.

Pyatkin [20] proved that if a $(3,4)$-biregular bipartite graph has a 3-regular subgraph covering the vertices of degree 4 , then it has an interval coloring. In $[1,8]$ another sufficient condition for the existence of an interval coloring of a $(3,4)$-biregular bipartite graph $G$ was obtained: $G$ admits an interval coloring if it has a spanning subgraph $F$, every component of which is a nontrivial path with endpoints at 3 -valent vertices and of length not exceeding 8. We call this a proper path factor of $G$. It was conjectured in [1] that every simple $(3,4)$-biregular bipartite graph has a proper path factor. (3,4)-biregular bipartite graphs with multiple edges need not have proper path factors, as shown in $[1,8]$.

In general, a path factor of a graph $G$ is a spanning subgraph of $G$ whose components are nontrivial paths. In [2] Asratian and Casselgren showed that every simple $(3,4)$ biregular bipartite graph has a path factor $F$ such that the endpoints of each path are at vertices of degree 3. However, no upper bound on the lengths of the paths in $F$ was established. In this note we present a variant of an algorithm introduced in [2] and show, using this algorithm, that there is an absolute constant $k$, such that every simple $(3,4)$ biregular bipartite graph has a path factor consisting of paths with endpoints at 3-valent vertices and of maximum length $k$. To be more precise, we prove the following theorem, which provides some further evidence for the conjecture in [1].

Theorem 1.1. Every simple (3,4)-biregular bipartite graph has a path factor $F$ where the endpoints of each path are at 3-valent vertices, and the length of each path in $F$ is at most 22 . 


\section{$2 \quad$ Proof of Theorem 1.1}

We denote by $d_{G}(v)$ the degree of a vertex $v$ in $G$ and by $N_{G}(v)$ the neighborhood of $v$ in $G$. For a subset $V^{\prime} \subseteq V(G)$, we set $N_{G}\left(V^{\prime}\right)=\cup_{v \in V^{\prime}} N_{G}(v)$. For any integer $n \geq 1$, the complete bipartite graph $K_{1, n}$ is called a star. A pseudo path factor of a bipartite graph $G$ with bipartition $(X, Y)$, where the vertices in $Y$ all have degree four, is a spanning subgraph $F$ of $G$ such that every component of $F$ is a path (possibly of length 0 ) and $d_{F}(y)=2$ for every $y \in Y$.

In [2] the author and Asratian proved that a pseudo path factor $F$ of a simple $(3,4)$ biregular bipartite graph $G$ can be transformed into a path factor $F^{\prime}$ of $G$, such that the endpoints of each path in $F^{\prime}$ have degree three in $G$ and no path in $F^{\prime}$ is longer than the longest path in $F$ (this is done by augmenting $F$ along trails with edges alternately in $F$ and $G-E(F)$, see Theorem 4 in [2]). This implies that instead of proving Theorem 1.1 we may show the following theorem, the proof of which will take up the rest of this section.

Theorem 2.1. Every simple (3,4)-biregular bipartite graph has a pseudo path factor such that the length of each path is at most 22 .

We need the following easy lemma.

Lemma 2.2. If a loop-free graph $G$ has no isolated vertices, then there is an edge cover $M$ of $G$, such that every component of $G[M]$ is a star.

Proof. Let $M^{\prime}$ be a maximum matching in $G$. Clearly, all vertices that are unsaturated by $M^{\prime}$ are nonadjacent. Furthermore, if $u v \in M^{\prime}$, then $u$ and $v$ cannot be adjacent to different unsaturated vertices, because then there is an $M^{\prime}$-augmenting path in $G$. We can thus extend $M^{\prime}$ to an edge cover $M$ by adding to $M^{\prime}$ an edge incident with each unsaturated vertex.

Proof of Theorem 2.1. Let $G$ be a $(3,4)$-biregular bipartite graph with bipartition $(X, Y)$ and let $Y^{\prime}$ be a maximum subset of $Y$, such that the vertices in $Y^{\prime}$ have disjoint neighborhoods in $G$. Note that every vertex $y \in Y \backslash Y^{\prime}$ is adjacent to at least one vertex of degree 2 in $G-Y^{\prime}$, because otherwise $y$ would have been included in $Y^{\prime}$. Let $Y_{3}$ be the set of vertices in $Y \backslash Y^{\prime}$ that are adjacent to exactly three vertices of degree 3 in $G-Y^{\prime}$. Remove from $G-Y^{\prime}$ a maximum subset $Y^{\prime \prime} \subseteq Y_{3}$ of vertices with disjoint neighborhoods in $G$ and denote the obtained graph by $H$. Let $H$ have bipartition $\left(X, Y_{H}\right)$, where $Y_{H}=Y \backslash\left(Y^{\prime} \cup Y^{\prime \prime}\right)$. We set $\hat{Y}=Y^{\prime} \cup Y^{\prime \prime}$ and define a new graph $I$ by setting $I=G[\hat{Y} \cup X]$. We need some properties of the graphs $H$ and $I$.

Claim 2.3. Every vertex in $Y_{H}$ is adjacent to at least two vertices of degree at most 2 in $H$, or adjacent to at least one vertex of degree 1 in $H$.

This is evident, since if a vertex $y \in Y_{H}$ does not satisfy the conditions of Claim 2.3, then it belongs to $Y_{3}$ and would have been included in $Y^{\prime \prime}$.

Claim 2.4. The graph I is a forest, every component of which is either 
(i) an isolated vertex that belongs to $X$,

(ii) a star consisting of one vertex $y \in \hat{Y}$ with four neighbors in $X$, or

(iii) a subgraph consisting of two vertices $y, y^{\prime} \in \hat{Y}$ with $\left|N_{I}\left(\left\{y, y^{\prime}\right\}\right)\right|=7$, and $\left|N_{I}(y) \cap N_{I}\left(y^{\prime}\right)\right|=1$.

Proof. Since $Y^{\prime \prime} \subseteq Y_{3}$, if $v \in Y^{\prime \prime}$, then there is exactly one vertex $u \in Y^{\prime}$, such that $u$ and $v$ have a common neighbor and, clearly, $\left|N_{G}(u) \cap N_{G}(v)\right|=1$. Furthermore, if $w \in Y^{\prime}$, then there cannot be two vertices $y, y^{\prime} \in Y^{\prime \prime}$, such that

$$
N_{G}(w) \cap N_{G}(y) \neq \emptyset \text { and } N_{G}(w) \cap N_{G}\left(y^{\prime}\right) \neq \emptyset,
$$

because this would contradict the maximality of $Y^{\prime}$. Thus a component in $I$ contains at most two vertices from $\hat{Y}$.

The proof of the next lemma is based on a variant of an algorithm introduced in [2] and is postponed to the next section.

Lemma 2.5. $H$ has a pseudo path factor $F$ such that the length of each path in $F$ does not exceed 4 , and for each vertex $x \in X$ with $d_{H}(x) \leq 2$, we have $d_{F}(x) \leq 1$.

Consider now the graph $I$ defined above. By Claim 2.4, I might contain a component with two vertices from $\hat{Y}$. For each component of $I$ with two vertices from $\hat{Y}$, remove exactly one of the edges incident with the common neighbor of the vertices in $\hat{Y}$, and denote the obtained graph by $I^{\prime}$. Suppose now that $F$ is a pseudo path factor of $H$ that satisfies the conditions of Lemma 2.5. Since $F$ is a pseudo path factor, the endpoints of all paths in $F$ are in $X$. If $x \in X$ and $d_{I^{\prime}}(x)>0$, then $d_{H}(x) \leq 2$ and Lemma 2.5 yields that $x$ is either an endpoint of a path in $F$ or is not in any nontrivial path in $F$. We construct from $I^{\prime}$ a new graph $J$ by setting $J=I^{\prime}+E^{\prime}$, where $E^{\prime}$ is a set of edges defined as follows: $e=x x^{\prime} \in E^{\prime}$ if and only if $x$ and $x^{\prime}$ are distinct endpoints of one path in $F$.

In the remaining part of the proof of Theorem 2.1 we will describe a method for constructing a subgraph $Q$ of $J$, such that every component of $Q$ is a path and $d_{Q}(y)=2$ for every vertex $y \in \hat{Y}$. Additionally, we require that $E^{\prime} \subseteq E(Q)$. Since the edges in $E^{\prime}$ correspond to paths in a pseudo path factor $F$ of $H$, the graph $Q$ will correspond to a pseudo path factor in $G$. The construction of $Q$ will be carried out in several steps.

We construct a new graph $K$ from $J$ by contracting every component of $I^{\prime}$ into a single vertex and removing all possibly arising loops but retaining multiple edges (if a component of $I^{\prime}$ already consists of a single vertex, then this vertex is included in $K$ without any changes). Thus two vertices $v, v^{\prime} \in V(K)$ are adjacent if and only if two vertices of the corresponding components of $I^{\prime}$ are adjacent in $J$, that is, if they are joined by an edge from $E^{\prime}$. Let $K^{\prime}$ be the graph obtained from $K$ by removing all isolated vertices. By Lemma 2.2, there is an edge cover $M$ in $K^{\prime}$ such that every component of $K^{\prime}[M]$ is a star. Denote by $M_{J}$ the edges in $J$ corresponding to the edges of $M$. Let $M_{I}$ be the set of edges $e \in E\left(I^{\prime}\right)$ such that $e$ is adjacent to an edge from $M_{J}$. 
Suppose that there is some vertex $y \in \hat{Y}$ that is not incident with any edge in $M_{I}$. Then, if $A$ is the component of $I^{\prime}$ containing $y$, we must have that the vertex in $K$ corresponding to $A$ is isolated. Consequently, if $e \in E^{\prime}$ and $e$ is incident with some vertex of $A$, then both ends of $e$ is in $A$. Since $d_{J}(y) \geq 3$, we may select two edges $e_{1}, e_{2} \in E(J)$ incident with $y$, such that if $e_{1}$ is adjacent to some edge $e^{\prime} \in E^{\prime}$, then $e_{2}$ is not adjacent to $e^{\prime}$. (If no edges of $E^{\prime}$ are adjacent to edges in $A$, then two arbitrary edges of $A$ incident with $y$ are selected.) By repeating this procedure for every vertex $y \in \hat{Y}$ that is not incident with any edge from $M_{I}$, we obtain a set of edges $E_{0}$. Let $E_{0}^{\prime}$ be the set of edges $e^{\prime} \in E^{\prime}$ such that $e^{\prime}$ is adjacent to an edge from $E_{0}$. Next, we define a set of edges $M_{I}^{\prime} \subseteq M_{I}$ as follows: For every vertex $y \in \hat{Y}$ that is incident with at least one edge of $M_{I}$, if more than two edges of $M_{I}$ are incident with $y$, then only two of those edges belong to $M_{I}^{\prime}$ (which ones do not matter), otherwise all edges of $M_{I}$ incident to $y$ belong to $M_{I}^{\prime}$. We now define a subgraph $Q_{0}$ of $J$ by setting

$$
V\left(Q_{0}\right)=V(J) \text { and } E\left(Q_{0}\right)=M_{J} \cup M_{I}^{\prime} \cup E_{0} \cup E_{0}^{\prime} .
$$

It follows from the construction of $Q_{0}$ that it satisfies the following conditions:

(a) $d_{Q_{0}}(y) \geq 1$ for each vertex $y \in \hat{Y}$;

(b) every component of $Q_{0}$ is a path of length not exceeding 6;

(c) every component of $Q_{0}$ contains at most three vertices from $\hat{Y}$, at most four vertices from $X$, and at most two edges from $E^{\prime}$;

(d) if $y \in \hat{Y}$ and $d_{Q_{0}}(y)=1$, then there are at least two edges $e_{1}, e_{2} \in E(J) \backslash E\left(Q_{0}\right)$ that are incident with $y$, and if $e^{\prime} \in E^{\prime}$ and $e^{\prime}$ is adjacent to $e_{1}$ or $e_{2}$, then $e^{\prime} \notin E\left(Q_{0}\right)$.

(e) if $e^{\prime} \in E^{\prime}$ and $e^{\prime}$ is adjacent to distinct edges $e_{1}, e_{2} \in E\left(I^{\prime}\right)$, then $e^{\prime} \in E\left(Q_{0}\right)$ if and only if $\left\{e_{1}, e_{2}\right\} \cap E\left(Q_{0}\right) \neq \emptyset$.

Let $\hat{Y}_{2}$ be the set of vertices $y \in \hat{Y}$ such that $d_{Q_{0}}(y)=2$, let $X_{1}$ be the vertices $x \in X$ such that $d_{Q_{0}}(x) \geq 1$, and define the graph $L$ by setting $L=J-\hat{Y}_{2}-X_{1}$. Next, let $L^{\prime}$ be the graph obtained from $L$ by contracting all edges in $E^{\prime} \cap E(L)$ and removing all loops but retaining multiple edges in the resulting graph. Note that $L^{\prime}$ is bipartite and $\hat{Y} \backslash \hat{Y}_{2}$ is one part in the bipartition.

Claim 2.6. There is a matching in $L^{\prime}$ that saturates every vertex in $V\left(L^{\prime}\right) \cap \hat{Y}$.

Proof. It is easily seen that if $x \in V\left(L^{\prime}\right) \backslash \hat{Y}$, then $d_{L^{\prime}}(x) \leq 2$. Let $y \in \hat{Y}$ with $d_{Q_{0}}(y)=1$. It follows from condition (d) and (e) that $d_{L^{\prime}}(y) \geq 2$. Moreover, if $\left|N_{L^{\prime}}(y)\right|=1$, then $y$ is the only neighbor of the vertex in $N_{L^{\prime}}(y)$. Thus, by Hall's condition there is a matching in $L^{\prime}$ that saturates all vertices in $V\left(L^{\prime}\right) \cap \hat{Y}$.

Let $R$ be a matching in $L^{\prime}$ that saturates every vertex in $V\left(L^{\prime}\right) \cap \hat{Y}$. Denote by $R_{J}$ the edges in $J$ corresponding to edges in $R$. By the construction of $L^{\prime}$, each edge of $E^{\prime}$ is 
adjacent to at most one edge of $R_{J}$ in $J$. It follows from this and conditions (a), (b), (d), (e) that the graph

$$
Q=Q_{0}+R_{J}+E^{\prime} \backslash E\left(Q_{0}\right)
$$

is a subgraph of $J$ such that $d_{Q}(y)=2$ for every $y \in \hat{Y}$, and every component of $Q$ is a path of length at most 10. If an endpoint of a path in $Q_{0}$ belongs to $\hat{Y}$, then the above construction of $Q$ adds one or two edges (in the latter case one of these edges belong to $\left.E^{\prime}\right)$ to this endpoint.

To sum up, every component of $Q$ is a path, $d_{Q}(y)=2$ for every vertex $y \in \hat{Y}$, the length of a path in $Q$ is at most $10, E^{\prime} \subseteq E(Q)$ and each path in $Q$ contains at most four edges from $E^{\prime}$. Since the edges of $E^{\prime}$ correspond to paths of length at most 4 in a pseudo path factor of $H, Q$ induces a pseudo path factor $P$ in $G$, such that the length of each path in $P$ does not exceed 22. We have thus proved Theorem 2.1.

\section{Proof of Lemma 2.5}

We prove Lemma 2.5 by presenting below an algorithm for constructing a pseudo path factor $F$ of $H$, such that the length of each path in $F$ is at most 4 and for each vertex $x \in X$ with $d_{H}(x) \leq 2$, we have $d_{F}(x) \leq 1$.

The algorithm constructs a sequence of subgraphs $F_{0}, F_{1}, F_{2}, \ldots$ of $H$, where $V\left(F_{0}\right)=$ $V(H), \emptyset=E\left(F_{0}\right) \subset E\left(F_{1}\right) \subset E\left(F_{2}\right) \subset \ldots$ and each component of $F_{p}$ is a path, for every $p \geq 1$. At each step $i \geq 1$ the algorithm constructs $F_{i}$ by adding to $F_{i-1}$ one or two edges until the condition $d_{F_{j}}(y)=2$ holds for all $y \in Y_{H}$, where $j \geq 1$. Then $F=F_{j}$ is a pseudo path factor of $H$. Parallelly the algorithm constructs a sequence of subgraphs $U_{0}, U_{1}, U_{2}, \ldots$ of $H$, where $V\left(U_{0}\right)=V(H), \emptyset=E\left(U_{0}\right) \subset E\left(U_{1}\right) \subset E\left(U_{2}\right) \subset \cdots \subset E\left(U_{j}\right)$. The edges of each $U_{i}$ will not be in the final pseudo path factor $F$.

The algorithm is based on Properties 3.1-3.6. During the execution of the algorithm the vertices in the set $X$ are considered to be unscanned or scanned. Initially all vertices in $X$ are unscanned. At the beginning of each step $i \geq 1$ we have a current vertex $y_{i} \in Y_{H}$. The algorithm selects a vertex $x_{i}$ among the unscanned vertices that are adjacent to $y_{i}$, and then determines which edges incident with $x_{i}$ will be in $F_{i}$ and which ones in $U_{i}$. If $d_{F_{i}}(v)=2$ for each $v \in Y_{H}$, then the algorithm stops. Otherwise the algorithm selects a new current vertex and goes to the next step.

\section{Algorithm}

Initially $F_{0}=(V(H), \emptyset), U_{0}=(V(H), \emptyset)$ and all vertices in $X$ are unscanned.

Step 0. Select a vertex $x_{0} \in X$ with $d_{H}\left(x_{0}\right)=2$. Let $y_{0}, y_{1}$ be the vertices in $Y_{H}$ adjacent to $x_{0}$ in $H$. Put $F_{1}=F_{0}+y_{0} x_{0}$ and $U_{1}=U_{0}+x_{0} y_{1}$. Consider the vertex $x_{0}$ to be scanned. Go to step 1 and let $y_{1}$ be the current vertex for step 1 . 
Step $i(i \geq 1)$. Step $i$ consists of two parts: the main part, where $F_{i}$ and $U_{i}$ are constructed, and the final part, where the current vertex for the next step is selected.

Main Part. Suppose that a vertex $y_{i}$ with $d_{F_{i-1}}\left(y_{i}\right) \leq 1$ was selected at step $(i-1)$ as the current vertex. By Property 3.4 (see below), $d_{U_{i-1}}\left(y_{i}\right) \leq 2$.

Therefore, there is an edge $x_{i} y_{i}$ with $x_{i} \in X$, such that $x_{i} y_{i} \notin E\left(F_{i-1}\right) \cup E\left(U_{i-1}\right)$. Moreover, if there is no edge $y_{i} x \in E\left(F_{i-1}\right)$ such that $d_{F_{i-1}}(x)=1$, then we choose $x_{i}$ so that $d_{H}\left(x_{i}\right) \leq 2$. By Property 3.5 we can make such a choice. In any case, the vertex $x_{i}$ is by Property 3.2 unscanned and therefore the subgraph $F_{i-1}+x_{i} y_{i}$ does not contain a cycle.

Since $d_{H}\left(x_{i}\right) \in\{1,2,3\}$, the vertex $x_{i}$ is, besides $y_{i}$, adjacent to either no vertex (Case 1 ), one vertex $w_{1}^{(i)}$ (Case 2-3) or two other vertices, $w_{1}^{(i)}$ and $w_{2}^{(i)}$ (Case 4-6).

Case 1. $d_{H}\left(x_{i}\right)=1$ :

Put $F_{i}=F_{i-1}+x_{i} y_{i}$ and consider the vertex $x_{i}$ to be scanned.

Case 2. $d_{H}\left(x_{i}\right)=2$ and $d_{F_{i-1}}\left(w_{1}^{(i)}\right)=2$ :

Put $F_{i}=F_{i-1}+x_{i} y_{i}$ and $U_{i}=U_{i-1}+x_{i} w_{1}^{(i)}$. Consider the vertex $x_{i}$ to be scanned.

Case 3. $d_{H}\left(x_{i}\right)=2$ and $d_{F_{i-1}}\left(w_{1}^{(i)}\right) \leq 1$ :

Put $F_{i}=F_{i-1}+x_{i} y_{i}$ and $U_{i}=U_{i-1}+x_{i} w_{1}^{(i)}$. Consider the vertex $x_{i}$ to be scanned.

Case 4. $d_{H}\left(x_{i}\right)=3$ and $d_{F_{i-1}}\left(w_{1}^{(i)}\right)=d_{F_{i-1}}\left(w_{2}^{(i)}\right)=2$ :

Put $F_{i}=F_{i-1}+x_{i} y_{i}$ and $U_{i}=U_{i-1}+\left\{x_{i} w_{1}^{(i)}, x_{i} w_{2}^{(i)}\right\}$. Consider the vertex $x_{i}$ to be scanned.

Case 5. $d_{H}\left(x_{i}\right)=3, d_{F_{i-1}}\left(w_{1}^{(i)}\right) \leq 1$ and $d_{F_{i-1}}\left(w_{2}^{(i)}\right)=2$ :

Put $F_{i}=F_{i-1}+x_{i} y_{i}, U_{i}=U_{i-1}+\left\{x_{i} w_{1}^{(i)}, x_{i} w_{2}^{(i)}\right\}$ and consider the vertex $x_{i}$ to be scanned.

Case 6. $d_{F_{i-1}}\left(w_{1}^{(i)}\right) \leq 1$ and $d_{F_{i-1}}\left(w_{2}^{(i)}\right) \leq 1$ :

Subcase 6a. $d_{F_{i-1}}\left(w_{1}^{(i)}\right)=0$ or $d_{F_{i-1}}\left(w_{2}^{(i)}\right)=0$ :

We assume that $d_{F_{i-1}}\left(w_{2}^{(i)}\right)=0$. Put $F_{i}=F_{i-1}+\left\{x_{i} y_{i}, x_{i} w_{2}^{(i)}\right\}, U_{i}=U_{i-1}+x_{i} w_{1}^{(i)}$ and consider the vertex $x_{i}$ to be scanned.

Subcase 6 b. $d_{F_{i-1}}\left(w_{1}^{(i)}\right)=d_{F_{i-1}}\left(w_{2}^{(i)}\right)=1$ and there is a vertex $x \in N_{F_{i-1}}\left(w_{1}^{(i)}\right) \cup N_{F_{i-1}}\left(w_{2}^{(i)}\right)$ such that $d_{F_{i-1}}(x)=1$ :

We assume that $w_{2}^{(i)}$ is adjacent (in $F_{i-1}$ ) to a vertex of degree 1 in $F_{i-1}$. Put $F_{i}=F_{i-1}+\left\{y_{i} x_{i}, x_{i} w_{2}^{(i)}\right\}, U_{i}=U_{i-1}+x_{i} w_{1}^{(i)}$ and consider the vertex $x_{i}$ to be scanned.

Subcase 6c. $d_{F_{i-1}}\left(w_{1}^{(i)}\right)=d_{F_{i-1}}\left(w_{2}^{(i)}\right)=1$ and there is no vertex $x \in N_{F_{i-1}}\left(w_{1}^{(i)}\right) \cup N_{F_{i-1}}\left(w_{2}^{(i)}\right)$ such that $d_{F_{i-1}}(x)=1$ :

By Property 3.6, we have $d_{U_{i-1}}\left(w_{1}^{(i)}\right)=d_{U_{i-1}}\left(w_{2}^{(i)}\right)=0$ or at least one of the vertices $w_{1}^{(i)}$ and $w_{2}^{(i)}$ is adjacent to a vertex of degree 1 in $H$. 
First suppose that $d_{U_{i-1}}\left(w_{1}^{(i)}\right)=d_{U_{i-1}}\left(w_{2}^{(i)}\right)=0$. Put $F_{i}=F_{i-1}+x_{i} y_{i}$, $U_{i}=U_{i-1}+\left\{x_{i} w_{1}^{(i)}, x_{i} w_{2}^{(i)}\right\}$ and consider the vertex $x_{i}$ to be scanned.

Now suppose instead that $w_{2}^{(i)}$ is adjacent to a vertex $u_{1}$ with $d_{H}\left(u_{1}\right)=1$. By our assumption about $w_{2}^{(i)}$ and since $d_{H}\left(u_{1}\right)=1$, the vertex $u_{1}$ is certainly unscanned. Put $F_{i}=F_{i-1}+\left\{y_{i} x_{i}, w_{2}^{(i)} u_{1}\right\}, U_{i}=U_{i-1}+\left\{x_{i} w_{1}^{(i)}, x_{i} w_{2}^{(i)}\right\}$ and consider the vertices $x_{i}, u_{1}$ to be scanned.

Final Part. In Cases 1, 2, 4 above, we select the current vertex for the next step according to the following rule: If $d_{F_{i}}(v)=2$ for every vertex $v \in Y_{H}$ then Stop. Otherwise select an arbitrary vertex $y_{i+1} \in Y_{H}$ with $d_{F_{i}}\left(y_{i+1}\right) \leq 1$, go to step $(i+1)$ and consider $y_{i+1}$ as the current vertex for step $(i+1)$.

If Case 3,5 or 6 above applies, then we put $y_{i+1}=w_{1}^{(i)}$, go to step $(i+1)$ and consider $y_{i+1}$ as the current vertex for step $(i+1)$.

Now we will prove the correctness of the algorithm. At the beginning of step $i$ we have that $y_{i}$ is the current vertex and the algorithm selects an unscanned vertex $x_{i}$ adjacent to $y_{i}$. If $d_{H}\left(x_{i}\right)=2$ then $w_{1}^{(i)}$ is the other vertex adjacent to $x_{i}$, if $d_{H}\left(x_{i}\right)=3$ then another vertex $w_{2}^{(i)}$ is also adjacent to $x_{i}$. The following property is evident.

Property 3.1. The algorithm determines which edges incident with $x_{i}$ will be in $F_{i}$ and which edges will be in $U_{i}$, the edge $y_{i} x_{i}$ will always be included in $F_{i}$. The vertex $x_{i}$ is then considered to be scanned and the algorithm will never consider $x_{i}$ again. If $d_{H}\left(x_{i}\right) \leq 2$, then only one edge incident with $x_{i}$ will be included in $F_{i}$.

The next property follows from Property 3.1.

Property 3.2. If $x \in X, y \in Y_{H}$ and the edge $x y \notin E\left(F_{i-1}\right) \cup E\left(U_{i-1}\right)$, then the vertex $x$ is unscanned at the beginning of step $i$.

It is straightforward to verify that the next property follows from the description of the algorithm.

Property 3.3. If an edge incident with a vertex $y \in Y_{H}$ is included in $U_{l}$ at step $l$ and $d_{F_{l}}(y)<d_{U_{l}}(y)$, then $y$ will be the current vertex of step $(l+1)$.

Suppose now that $d_{U_{l-1}}(y)=d_{F_{l-1}}(y)=0$ and an edge $e$ incident with $y \in Y_{H}$ is included in $U_{l}$ at step $l$. It follows from Property 3.3 that $y$ will be the current vertex of step $(l+1)$ and according to the description of the algorithm, a vertex $x_{l+1} \in N_{H}(y)$ such that $x_{l+1} y \notin E\left(F_{l}\right) \cup E\left(U_{l}\right)$ would be selected at step $(l+1)$. The vertex $x_{l+1}$ is by Property 3.2 unscanned at step $(l+1)$ and Property 3.1 yields that $y x_{l+1}$ would be included in $F_{l+1}$ and we would have $d_{F_{l+1}}(y)=d_{U_{l+1}}(y)=1$.

Similarly, if yet another edge $e^{\prime}$ incident with $y$ is included in $U_{m}$ at step $m>l$, then either another edge $e^{\prime \prime} \notin E\left(F_{m-1}\right) \cup E\left(U_{m-1}\right)$, incident with $y$, would be included in $F_{m}$ at step $m$ (Subcase 6c above), or, by Property 3.3, $y$ would be the current vertex of step 
$(m+1)$. In the latter case, $d_{U_{m}}(y)=2$ and $d_{F_{m}}(y)=1$ and since $d_{H}(y)=4$, there is a vertex $x_{m+1} \in N_{H}(y)$ such that $x_{m+1} y \notin E\left(F_{m}\right) \cup E\left(U_{m}\right)$. Then $x_{m+1}$ is unscanned at step $(m+1)$ and by Property 3.1, $x_{m+1} y$ would be included in $F_{m+1}$ at step $(m+1)$. Thus we have the following property.

Property 3.4. If $y \in Y_{H}$ and $d_{F_{i-1}}(y)=0$, then $d_{U_{i-1}}(y) \leq 1$, and if $y \in Y_{H}$ and $d_{F_{i-1}}(y)=1$, then $d_{U_{i-1}}(y) \leq 2$.

Property 3.5. If the current vertex $y_{i}$ of step $i$ is not incident with an edge $y_{i} x \in E\left(F_{i-1}\right)$ such that $d_{F_{i-1}}(x)=1$, then $y_{i}$ is adjacent to a vertex $x_{i}$ with $d_{H}\left(x_{i}\right) \leq 2$, such that $y_{i} x_{i} \notin E\left(F_{i-1}\right) \cup E\left(U_{i-1}\right)$.

Proof. Let $y_{i}$ be the current vertex of step $i$ and assume that there is no edge $y_{i} x \in$ $E\left(F_{i-1}\right)$, such that $d_{F_{i-1}}(x)=1$. Suppose further that there is no vertex $x_{i}$ adjacent to $y_{i}$ such that $y_{i} x_{i} \notin E\left(F_{i-1}\right) \cup E\left(U_{i-1}\right)$ and $d_{H}\left(x_{i}\right) \leq 2$.

By Claim 2.3, $y_{i}$ is adjacent to at least two vertices of degree at most 2 in $H$ or a vertex of degree 1 in $H$. In the latter case, the desired result follows, because if there is such a vertex $u$, then by Property 3.1, either $u y_{i} \in E\left(F_{i-1}\right)$ or $u$ is unscanned. So assume that $y_{i}$ is not adjacent to a vertex of degree 1 in $H$, and let $x_{i_{1}}, x_{i_{2}}$ be the two vertices in $N_{H}\left(y_{i}\right)$ that satisfy $d_{H}\left(x_{i_{1}}\right)=d_{H}\left(x_{i_{2}}\right)=2$. Since we assumed that there was no vertex $x_{i}$, such that $d_{H}\left(x_{i}\right)=2$ and $y_{i} x_{i} \notin E\left(F_{i-1}\right) \cup E\left(U_{i-1}\right)$, the vertices $x_{i_{1}}, x_{i_{2}}$ must be scanned at step $i$. Property 3.1 yields that $d_{F_{i-1}}\left(x_{i_{1}}\right) \leq 1, d_{F_{i-1}}\left(x_{i_{2}}\right) \leq 1$ and we thus have $\left\{y_{i} x_{i_{1}}, y_{i} x_{i_{2}}\right\} \subseteq E\left(U_{i-1}\right)$.

Let $j$ be the minimum integer such that $j \leq i-1$ and one of the edges $y_{i} x_{i_{1}}, y_{i} x_{i_{2}}$ was included in $U_{j}$ at step $j$. Suppose that $y_{i} x_{i_{1}}$ was included in $U_{j}$ at step $j$. Then by the description of the algorithm (Case 3 above), $y_{i}$ would be the current vertex of step $(j+1)$. Since $y_{i} x_{i_{2}} \notin E\left(U_{j}\right)$, we must have $j<i-1$. Moreover, since $x_{i_{2}}$ is unscanned at step $(j+1), y_{i} x_{i_{2}}$ would have been included in $F_{j+1}$ at step $(j+1)$. It follows from Property 3.1 that $d_{F_{j+1}}\left(x_{i_{2}}\right)=1$, because $d_{H}\left(x_{i_{2}}\right)=2$. Since $j+1 \leq i-1, F_{j+1} \subseteq F_{i-1}$, and this is contradiction to our assumption above.

Property 3.6. Let $x \in X$ be an unscanned vertex at the beginning of step $i$ that satisfies $d_{H}(x)=3$. Suppose that $x$ is adjacent to a vertex $y \in Y_{H}$ such that

(i) $d_{F_{i-1}}(y)=1$

(ii) $y$ is not incident with an edge $u y \in E\left(F_{i-1}\right)$, such that $d_{F_{i-1}}(u)=1$,

(iii) $y$ is not the current vertex of step $i$.

Then $d_{U_{i-1}}(y)=0$ or $y$ is adjacent to a vertex $w$ with $d_{H}(w)=1$.

Proof. Suppose that $x$ and $y$ are vertices that satisfy the hypothesis of Property 3.6. Then there is a vertex $x^{\prime} \in N_{F_{i-1}}(y)$ such that $d_{F_{i-1}}\left(x^{\prime}\right)=2$. Property 3.1 implies that $d_{H}\left(x^{\prime}\right)=3$. Suppose that $y$ is not adjacent to a vertex $w \in X$ with $d_{H}(w)=1$. Then, since $d_{H}(x)=d_{H}\left(x^{\prime}\right)=3$, it follows from Claim 2.3 that the other two vertices $x_{i_{1}}, x_{i_{2}}$ in $N_{H}(y)$ satisfy $d_{H}\left(x_{i_{1}}\right)=d_{H}\left(x_{i_{2}}\right)=2$. 
Suppose, for a contradiction, that $d_{U_{i-1}}(y)>0$. This implies that $x_{i_{1}} y \in E\left(U_{i-1}\right)$ or $x_{i_{2}} y \in E\left(U_{i-1}\right)$. Let $j$ be the minimum integer such that $j \leq i-1$ and one of the edges $y x_{i_{1}}$ and $y x_{i_{2}}$ was included in $U_{j}$ at step $j$. Suppose, for instance, that $y x_{i_{1}}$ was included in $U_{j}$ at step $j$. Then by the description of the algorithm (Case 3 above), $y$ would be the current vertex of step $(j+1)$. Since $y$ is not the current vertex of step $i$, we have that $j+1<i$, and since $y x_{i_{2}} \notin E\left(F_{j}\right) \cup E\left(U_{j}\right), x_{i_{2}}$ is unscanned at step $(j+1)$. This implies that $y x_{i_{2}}$ would be included in $F_{j+1}$ and by Property $3.1, d_{F_{j+1}}\left(x_{i_{2}}\right)=1$. Since $j+1<i$, $F_{j+1} \subseteq F_{i-1}$, and this is a contradiction.

It follows from the description of the algorithm and Properties 3.1-3.6 that the algorithm will stop at step $i$ only when $d_{F_{i}}(v)=2$ for every $v \in Y_{H}$, that is, when $F_{i}$ is a pseudo path factor of $H$. It is also clear that every vertex $y \in Y_{H}$ is adjacent to at least one vertex of degree 1 in the final pseudo path factor $F=F_{i}$ and therefore the length of each path in $F$ does not exceed 4. Additionally, by Property 3.1, if $x \in X$ and $d_{H}(x) \leq 2$, then $d_{F}(x) \leq 1$. The proof of Lemma 2.5 is complete.

\section{Concluding remarks}

In [1] several examples of simple $(3,4)$-biregular bipartite graphs having proper path factors using only paths of length 6 was presented. If a simple $(3,4)$-biregular bipartite graph has a cut vertex, then it need not have a proper path factor containing only paths of length 6 [8]. In addition to the conjecture made in [1] (every simple (3,4)-biregular bipartite graph has a proper path factor) we conjecture that every simple 2-connected $(3,4)$-biregular bipartite graph has a proper path factor containing only paths of length 6.

The following might also be worth noting: Let $G$ be a simple $(3,4)$-biregular bipartite graph with bipartition $(X, Y)$. If there is a subset $Y^{\prime} \subseteq Y$, such that the vertices of $Y^{\prime}$ have disjoint neigborhoods and every vertex in $Y \backslash Y^{\prime}$ is adjacent to at least two vertices of degree 2 in $G-Y^{\prime}$, then the method for constructing path factors presented here will construct a path factor $P$ of $G$, such that the endpoints of each path in $P$ have degree three and the length of each path in $P$ does not exceed 10. (In the proof of Theorem 2.1 the set $Y^{\prime \prime}$ will be empty, every component of $I$ contains only one vertex from $\hat{Y}$, and one can use a simpler method for constructing the subgraph $Q$ so that each path in $Q$ has length at most four and contains at most two edges from $E^{\prime}$.) It is, however, easy to find examples of simple $(3,4)$-biregular bipartite graphs that do not have this property. An example of such a graph $G$ with bipartition $(X, Y)$ can be constructed as follows: Let

$$
Y=\left\{y_{1}, y_{2}, y_{3}, y_{4}, y_{5}, y_{6}, y_{7}, y_{8}, y_{9}\right\} \text { and } X=\left\{x_{1}, x_{2}, x_{3}, x_{4}, x_{5}, x_{6}, x_{7}, x_{8}, x_{9}, x_{10}, x_{11}, x_{12}\right\} \text {, }
$$

where adjacency is defined by:

$$
\begin{aligned}
& N_{G}\left(x_{1}\right)=\left\{y_{1}, y_{2}, y_{3}\right\}, N_{G}\left(x_{2}\right)=\left\{y_{4}, y_{5}, y_{6}\right\}, N_{G}\left(x_{3}\right)=\left\{y_{7}, y_{8}, y_{9}\right\}, \\
& N_{G}\left(x_{4}\right)=\left\{y_{1}, y_{4}, y_{7}\right\}, N_{G}\left(x_{5}\right)=\left\{y_{2}, y_{5}, y_{8}\right\}, N_{G}\left(x_{6}\right)=\left\{y_{3}, y_{6}, y_{9}\right\},
\end{aligned}
$$




$$
\begin{aligned}
& N_{G}\left(x_{7}\right)=\left\{y_{1}, y_{5}, y_{9}\right\}, N_{G}\left(x_{8}\right)=\left\{y_{2}, y_{6}, y_{7}\right\}, N_{G}\left(x_{9}\right)=\left\{y_{3}, y_{4}, y_{8}\right\}, \\
& N_{G}\left(x_{10}\right)=\left\{y_{1}, y_{6}, y_{8}\right\}, N_{G}\left(x_{11}\right)=\left\{y_{2}, y_{4}, y_{9}\right\}, N_{G}\left(x_{12}\right)=\left\{y_{3}, y_{5}, y_{7}\right\} .
\end{aligned}
$$

For every two vertices $y_{i}, y_{j} \in Y$ there is a path of length 2 between $y_{i}$ and $y_{j}$. Hence, no two vertices of $Y$ have disjoint neighborhoods and clearly there is no vertex $y \in Y$ such that every vertex in $Y \backslash\{y\}$ is adjacent to two vertices of degree 2 in $G-y$.

\section{Acknowledgements}

We thank one of the referees for helpful comments and remarks.

\section{References}

[1] A. S. Asratian, C. J. Casselgren, Jennifer Vandenbussche, Douglas B. West, Proper path-factors and interval edge-coloring of $(3,4)$-biregular bigraphs, Journal of Graph Theory 61 (2009), 88-97.

[2] A. S. Asratian, C. J. Casselgren, On path factors of (3,4)-biregular bigraphs, Graphs and Combinatorics 24 (2008), 405-411.

[3] A. S. Asratian, C. J. Casselgren, On interval edge colorings of $(\alpha, \beta)$-biregular bipartite graphs, Discrete Mathematics 307 (2007), 1951-1956.

[4] A. S. Asratian, R. R. Kamalian, Interval coloring of the edges of a multigraph (in Russian), Applied Mathematics 5 (1987), 25-34, Yerevan University.

[5] A. S. Asratian, R. R. Kamalian, Investigation of interval edge-colorings of graphs, Journal of Combinatorial Theory, Series B 62 (1994), 34-43.

[6] M. A. Axenovich, On interval colorings of planar graphs, Congressus Numerantium 159 (2002), 77-94.

[7] J. A. Bondy, U. S. R. Murty, Graph theory with applications, American Elsevier Publishing Co., Inc., New York, 1976.

[8] C. J. Casselgren, Some results on interval edge colorings of bipartite graphs, Master's thesis, Linköping University, 2005.

[9] K. Giaro, The complexity of consecutive $\Delta$-coloring of bipartite graphs: 4 is easy, 5 is hard, Ars Combinatoria 47 (1997), 287-298.

[10] K. Giaro, M. Kubale, Compact scheduling of zero-one time operations in multi-stage systems, Discrete Applied Mathematics 145 (2004), 95-103

[11] K. Giaro, M. Kubale, Consecutive edge-colorings of complete and incomplete Cartesian products of graphs, Congressus Numerantium 128 (1997), 143-149.

[12] K. Giaro, M. Kubale, M. Malafiejski, Consecutive colorings of the edges of general graphs, Discrete Mathematics 236 (2001), 131-143.

[13] K. Giaro, M. Kubale, M. Malafiejski, On the deficiency of bipartite graphs, Discrete Applied Mathematics 94 (1999), 193-203 
[14] H. M. Hansen, Scheduling with minimum waiting periods (in Danish), Master's Thesis, Odense University, Odense, Denmark, 1992.

[15] D. Hanson, C. O. M. Loten, B. Toft, On interval colourings of bi-regular bipartite graphs, Ars Combinatoria 50 (1998), 23-32.

[16] D. Hanson, C. O. M. Loten, A lower bound for interval colouring bi-regular bipartite graphs, Bulletin of the ICA 18 (1996), 69-74.

[17] T. Jensen, B. Toft, Graph Coloring Problems, Wiley-Interscience, Wiley, New York, 1995.

[18] R. R. Kamalian, Interval edge-colorings of graphs, Doctoral thesis, Novosibirsk, 1990.

[19] A. V. Kostochka, Unpublished manuscript, 1995

[20] A. V. Pyatkin, Interval coloring of $(3,4)$-biregular bipartite graphs having large cubic subgraphs, Journal of Graph Theory 47 (2004), 122-128.

[21] S. V. Sevastjanov, Interval colorability of the edges of a bipartite graph (in Russian), Metody Diskretnogo Analiza 50 (1990), 61-72. 